\title{
1 Shallow-structure characterization by 2D elastic full-waveform inversion
}

\author{
2 Anouar Romdhane ${ }^{1}$, Gilles Grandjeany ${ }^{2}$, Romain Brossierx ${ }^{3}$, Faycal Rejibaz ${ }^{4}$, \\ Stephane Operto ${ }^{5}$, and Jean Virieux ${ }^{3}$
}



multifrequency inversion of damped data, to mitigate the strong nonlinearities coming from the surface waves. This strategy is a key point in alleviating the strong near-surface effects and avoiding convergence toward a local minimum. Using a limited-memory quasi-Newton method improved the convergence level. These findings are analogous to recent applications on large-scale domains, although limited sourcereceiver offset ranges, low-frequency content of the source, and domination of surface waves on the signal led to some difficulties. Regarding the impact of data decimation on the inversion results, we have learned that an inversion restricted to the vertical data component can be successful without significant loss in terms of parameter imagery resolution. In our investigations of the effect of increased source spacing, we found that a sampling of $4 \mathrm{~ms}$ (less than three times the theoretical maximum of one half-wavelength) led to severe aliasing.

\section{INTRODUCTION}

Accurate subsurface imaging based on seismic methods constitutes one of the main issues encountered in the environmental and civil engineering fields. It offers the possibility of taking advantage of a noninvasive technique to depict subsoil structures of the first $100 \mathrm{~m}$ as a reconstructed image from a seismic wavefield recorded at the surface. This can be achieved using several reconstruction techniques that analyze different kinds of waves associated with propagation phenomena (diffraction, reflection, dispersion, refraction, etc.).
The most conventional technique is based on the inversion of body-wave arrival traveltimes, particularly P-waves, using direct or refracted waves. Efficiency of the process closely depends on the realism of the associated forward problem to account for the characteristics of the medium (heterogeneities, contrasts) in calculating traveltimes. In this context, robustness and efficiency of the ray-tracing technique, based on the asymptotic ray theory in the high-frequency approximation, are restricted to the case of smoothed media (Červený et al., 1977; Červený, 2001) and consequently are unsuitable for highly heterogeneous subsurface domains.

Manuscript received by the Editor 24 February 2010; revised manuscript received 22 August 2010; published online 00000.

${ }^{1}$ Formerly BRMG, Orleans, France; presently Foundation for Scientific and Industrial Research (SINTEF), Trondheim, Norway. E-mail: anouar.romdhane@sintef.no.

${ }^{2}$ BRGM, Natural Risks and CO2 Storage Security Division, Orlęans, France. E-mail: g.grandjean@brgm.fr.

${ }^{3}$ Universite Joseph Fourier, Laboratoire de Geophysique Interne et Tectonophysique, Centre National de la Recherche Scientifique (CNRS), Institut de Recherche pour le Développement (IRD), Grenoble, France. E-mail: romain.brossier@obs.ujf-grenoble.fr; jean.virieux@obs.ujf-grenoble.fr.

${ }_{5}^{4}$ Universite_Pierre et Marie Curie, Paris, France. E-mail: faycal.rejiba@upmc.fr.

${ }^{5}$ Universite Nice-Sophia Antipolis, Geoazur, Centre National de la Recherche Scientifique (CNRS), Institut de Recherche pour le Développement (IRD), Observatoire de la Cette d'Azur, Villefranche-sur-mer, France. E-mail: operto@geoazur.obs-vlfr.fr.

(C) 2011 Society of Exploration Geophysicists. All rights reserved. 
An alternative to ray tracing and the more robust wavefront construction technique (Vinje et al., 1993, 1996a, 1996b) consists of applying finite differences to solve the eikonal equation numerically (Vidale, 1988; Podvin and Lecomte, 1991), making it possible to deal with more heterogenous media. Important progress has been achieved to handle the associated inverse problem efficiently, using the popular simultaneous iterative reconstruction technique (SIRT) (van der Sluis and van der Vorst, 1987; Grandjean and Sage, 2004) or the more appealing adjoint state method (Taillandier et al., 2009). However, applications to real data in the context of shallow prospecting (Grandjean and Leparoux, 2004; Ellefsen, 2009) reveal restrictions. This is particularly the case when later arrivals must be included in the inversion scheme or when dealing with real data where surface waves, which always represent the main component (about two-thirds) of the seismic energy, can seriously mitigate signals used in the inversion.

On the other hand, with the introduction of the spectral analysis of surface waves (SASW) method (Nazarian and Stokoe, 1984, 1986; Stokoe and Nazarian, 1985; Stokoe et al., 1988), surface waves have received much attention. The good signalto-noise ratio $(\mathrm{S} / \mathrm{N})$ of these waves associated with the relative ease of their acquisition gives rise to a variety of applications (Lai, 1998; Park et al., 1999; Rix et al., 2001). Early studies were devoted to reconstructing 1D shear-wave velocity distribution by calculating phase differences between two receivers. The SASW method was later extended to the multichannel analysis of surface waves (MASW), which is based on the phase-velocity variation with frequency from a multichannel recording system.

The 1D assumption of the MASW method is imposed by the formulation used for solving the inverse problem (Hermann, 1991). To overcome this limitation, some extensions have been made (Park et al., 1998; Xia et al., 1999; Grandjean and Bitri, 2006) to adapt the methodology to $2 \mathrm{D}$ contexts by narrowing offset windows or/and using a summation principle to increase the $\mathrm{S} / \mathrm{N}$. The resulting $1 \mathrm{D}$ velocity profiles are then interpolated along a seismic line to produce a 2D view of the shear velocity.

Despite all of these developments, some limitations still alter the potential of surface-wave methods. These limitations are mainly the result of difficulties encountered when identifying and separating the first (fundamental) propagation mode from higher modes (possible propagation modes of surface waves in a layered medium), which form the basis of the inversion process. This phenomenon, in addition to the errors resulting from fitting data including 2D or 3D effects (phase-velocity changes) under a 1D assumption, drastically mitigate the efficiency of the MASW method (Bodet, 2005). Some recent results have also shown that the dispersion curve is not an intrinsic property of the medium by emphasizing the influence of acquisition parameters (Socco and Strobia, 2004).

An important point is the common feature of MASW and first-break acoustic tomography. Both use a restrictive part of the information contained in the seismic signal: the dispersion of Rayleigh waves and the first P-wave arrivals. A strategy integrating both signals should be more efficient and physically consistent to reduce the possible solutions satisfying the approaches. To overcome this issue, an alternative approach consists of taking advantage of recent advances in quantitative imaging based on full-waveform inversion (FWI) in the time (Tarantola, 1984) or frequency domains (Pratt et al., 1998). In theory, these approaches offer important possibilities because they use al in 122 formation contained in seismic signals (P-waves in the acoustic 123 case and P-SV-SH-waves in the elastic case) in the inversion 124 strategy. The inverse problem formulation in the frequency do- 125 main has been implemented and applied to synthetic and real 126 data concerning large-scale domains (kilometric scale) (Ravaut 127 et al., 2004; Brenders and Pratt, 2007; Brossier et al., 2009). 128 This context is very different from the subsurface one because 129 low-frequency sources and long offsets can be used. Surface 130 waves can be separated easily from body waves and tradition- 131 ally are muted.

However, in the context of near-surface imaging, the greatest 133 part of the energy emitted by a surface seismic source contrib- 134 utes to the generation of surface waves. To overcome this limi- 135 tation, some workers have proposed applying a time window to 136 the early arrivals and performing acoustic waveform tomogra- 137 phy with near-surface data (Gao et al., 2006, 2007; Sheng et al., 138 2006; Smithyman et al., 2009). Results show that this strategy 139 outperforms traveltime tomography and is well suited to data 140 coming from refraction surveys where far and intermediate off- 141 sets are considered. However, when the offset range is too small 142 (which is usually the case in near-surface prospecting) to allow 143 separation between body waves and surface waves, the effi- 144 ciency of this strategy may be severely altered. Moreover, this 145 strategy does not take advantage of the information included in 146 shear and surface waves that are usually considered as a source 147 of noise in the inversion. These waves propagate with a lower 148 velocity than compressional waves and may therefore lead to 149 higher resolution of the images.

To our knowledge, only a few studies of waveform inversion 151 involving body and surface waves have been performed for 152 near-surface $(0-100 \mathrm{~m})$ investigations (Gélis et al., 2007; Romd- 153 hane et al., 2008). These numerical investigations were per- 154 formed to image near-surface heterogeneities with various con- 155 trasts in a well-known background medium. In these cases, 156 surface topography was considered to be flat, mainly because of 157 the computational difficulties encountered to model surface 158 waves accurately in the presence of a complex topography 159 (Moczo et al., 2007). The effects of irregular topography on 160 seismic wave motion have been the subject of some numerical 161 investigations (Bleibinhaus and Rondenay, 2009; Shiann-Jong 162 et al., 2009). It is well established that topography can drasti- 163 cally influence amplitudes and phases of the seismic signal. 164 Consequently, correct modeling of free-surface effects is a criti- 165 cal requirement for any seismic-inversion process.

Recent work conducted by Brossier et al. (2009) focuses on 167 the impact of applying several multiresolution strategies to miti- 168 gate the strong nonlinearity inherent in surface waves. Simula- 169 tions performed with a section of the well-known SEG/EAGE 170 overthrust model reveal that preconditioning provided by time 171 damping associated with successive inversions of overlapping 172 frequency groups is critical to converge toward acceptable ve- 173 locity models.

The objective of our work is thus to evaluate the effectiveness 175 of using an FWI algorithm to take advantage of the information 176 contained in surface waves to image heterogeneous shallow 177 structures in the context of a complex surface topography. Our 178 paper is organized in two sections. In the first section, we pres- 179 ent a brief review of the basis of the elastic FWI technique 180 used. In the second section, we apply it to a synthetic but 181 
realistic landslide case, derived from the structure of the SuperSauze earthflow. We evaluate the efficiency of using preconditioning strategies to reconstruct the shallow velocity structure. We also address the effects of some practical considerations, particularly the restriction to the vertical data component and the impact of acquisition decimation, typically related to subsurface prospecting, on inversion results.

\section{ELASTIC FULL-WAVEFORM INVERSION}

We first consider the 2D P-SV-wave modeling case. The forward and inverse problems are solved in the frequency domain. The forward problem is based on a discontinuous Galerkin (DG) approach. An FWI algorithm is used to solve the inverse problem. It is based on the preconditioned conjugate-gradient (PCG) method or a limited-memory quasi-Newton Broyden-FletcherGoldfarb-Shanno (L-BFGS) approach and is implemented on a parallel computation architecture. We then present the preconditioning strategies used for our numerical simulations.

\section{The forward problem}

In an isotropic elastic medium, the equation system governing the wave propagation in 2D media relates velocities $\partial V_{x}$ and $V_{z}$ to stresses $\sigma_{x x}, \sigma_{z z}$, and $\sigma_{x z}$. It can be written in the frequency domain as

$$
\begin{aligned}
-i \omega \rho V_{x} & =\frac{1}{\rho(\mathbf{x})}\left[\frac{\partial \sigma_{x x}}{\partial x}+\frac{\partial \sigma_{x z}}{\partial z}\right]+F_{x}, \\
-i \omega \rho V_{z} & =\frac{1}{\rho(\mathbf{x})}\left[\frac{\partial \sigma_{x z}}{\partial x}+\frac{\partial \sigma_{z z}}{\partial z}\right]+F_{z}, \\
-i \omega \sigma_{x x} & =(\lambda(\mathbf{x})+2 \mu(\mathbf{x}))+\lambda(\mathbf{x}) \frac{\partial V_{z}}{\partial z}, \\
-i \omega \sigma_{z z} & =\lambda(\mathbf{x}) \frac{\partial V_{x}}{\partial x}+(\lambda(\mathbf{x})+2 \mu(\mathbf{x})) \frac{\partial V_{z}}{\partial z}, \\
-i \omega \sigma_{x z} & =\mu(\mathbf{x})\left[\frac{\partial V_{x}}{\partial z}+\frac{\partial V_{z}}{\partial x}\right],
\end{aligned}
$$

where $\lambda$ and $\mu$ are the Lamé coefficients, $\mathbf{x}$ is spatial position, $\rho$ is density, and $\omega$ is angular frequency. The physical properties of the medium are supposed to be constant inside each cell, and central numerical fluxes are used. Details of the mixed DG interpolation orders $\mathrm{P} 0-\mathrm{P} 1$ formulation, used in this study, are provided in Brossier (2009).

System 1 can be written with respect to a linear matrix formalism for each frequency considered:

$$
\mathbf{A x}=\mathbf{s}
$$

where vector $\mathbf{x}$ denotes the unknowns, consisting of the particle velocities and stresses, $\mathbf{s}$ is the source term, and $\mathbf{A}$ is the impedance matrix. To solve the linear system resulting from discretizing equation 2, the impedance matrix is first factorized with an LU decomposition independent of the source term. Solutions for multiple sources (i.e., multiple right-hand-side terms) can then be obtained efficiently by forward and backward substitutions. Parallel factorization of the impedance matrix is performed using the MUMPS massively parallel direct solver package (MUMPS, 2009).

Some recent results reveal promising prospects for applying the DG method to elastic-wave propagation. The use of high orders of interpolation is especially appealing because they 223 allow unstructured meshes and thus offer the possibility of 224 locally adapting the mesh size to local medium parameters (h- 225 adaptive mesh). They also ensure high accuracy with a coarse 226 discretization of the medium (Dumbser and Käser, 2006). How- 227 ever, this coarse discretization may be inconsistent with the 228 expected resolution of the FWI, which necessitates a discretiza- 229 tion close to $\lambda / 4$ (Sourbier et al., 2009).

In this study, we use a lower order of interpolation. Applying 231 the DG method based on the lowest interpolation order (P0) 232 turns out to be very efficient, in terms of computational cost, in 233 comparison with classical finite-difference formulations in the 234 context of contrasted media and smooth surface topography 235 (Brossier et al., 2008). The accuracy is guaranteed with only 236 10-15 cells per minimum wavelength compared to the 60 grid 237 points necessary with the rotated second-order stencil and the 238 vacuum formalism (Saenger et al., 2000; Bohlen and Saenger, 239 2006). In addition, an interesting compromise between accuracy 240 of wavefield estimation and computational cost consists of using 241 the mixed P0-P1 DG interpolation to overcome some particular 242 restrictions related to topography complexity. The use of 243 unstructured meshes (for P1) ensures precise implementation of 244 the source term and accurate modeling of the complex topogra- 245 phy, taking into account the free-surface boundary conditions 246 (Brossier, 2010). In addition, it offers the possibility to adapt 247 mesh size to the local physical parameters. This property is of 248 great interest, especially in the context of near-surface modeling 249 with weathered zones (with very low velocities).

\section{The inverse problem}

251

In this section, we briefly review the principles of FWI. An 252 extensive overview of the method can be found in Virieux and 253 Operto (2009).

In the case of weighted least-squares linearized inversion, the 255 misfit function $E$ can be expressed (Tarantola, 1987) as

$$
E(\mathbf{m})=\frac{1}{2}\left(\mathbf{d}_{\mathrm{obs}}-\mathbf{d}_{\mathrm{cal}}\right)^{\dagger} \mathbf{S}_{d}^{\dagger} \mathbf{S}_{d}\left(\mathbf{d}_{\mathrm{obs}}-\mathbf{d}_{\mathrm{cal}}\right),
$$

where the dagger $\uparrow$ denotes the transpose conjugate; $\mathbf{d}_{\text {obs }}$ and 257 $\mathbf{d}_{\text {cal }}$ denote observed and calculated data (particle velocities), 258 respectively; $\Delta \mathbf{d}=\left(\mathbf{d}_{\mathrm{obs}}-\mathbf{d}_{\text {cal }}\right)$ corresponds to the data-misfit 259 vector in model $\mathbf{m}$; and $\mathbf{S}_{d}$ is a weighting operator applied to 260 the data. A solution to equation 3 is to linearize it in the second 261 order around an initial model $m_{l}$, which corresponds to the 262 model of the $l$ th iteration as follows:

$$
\begin{aligned}
E\left(\mathbf{m}^{(l-1)}+\delta \mathbf{m}^{(l)}\right)= & E\left(\mathbf{m}^{(l-1)}\right)+\nabla_{m} E\left(\mathbf{m}^{(l-1)}\right) \delta \mathbf{m}^{(l)} \\
& +\frac{1}{2} \delta \mathbf{m}^{(l) \dagger} H\left(\mathbf{m}^{(l-1)}\right) \delta \mathbf{m}^{(l)},
\end{aligned}
$$

where $\delta \mathbf{m}^{(l)}$ is the model perturbation and where $\nabla_{m} E\left(\mathbf{m}^{(l-1)}\right) 264$ and $H\left(\mathbf{m}^{(l-1)}\right)$ are the gradient and Hessian of the misfit function, 265 respectively. Minimizing $E$ leads to the Newton equation, which 266 relates the model perturbation to the gradient and Hessian as

$$
\delta \mathbf{m}^{(l)}=-\left[H\left(\mathbf{m}^{(l-1)}\right)\right]^{-1} \nabla_{m} E\left(\mathbf{m}^{(l-1)}\right) .
$$

The gradient direction is computed efficiently following the 268 adjoint-state formulation (Plessix, 2006). For one model parame- 269 ter $k$, the system can be recast in matrix form: 


$$
\nabla_{m} E\left(m_{k}\right)=\Re\left[\mathbf{x}^{t}\left[\frac{\delta \mathbf{A}}{\delta m_{k}}\right]^{t} \mathbf{A}^{-1} \tilde{\mathcal{P}} \mathbf{S}_{d}^{\dagger} \mathbf{S}_{d} \mathbf{d}^{*}\right],
$$

where $\tilde{\mathcal{P}}$ is an operator that projects the data residual vector in the data space to the model space, $\Re$ is the real part of a complex number, $\Delta \mathbf{d}$ corresponds to the data misfit vector, and $t$ and * are the transpose and conjugate operators. Equation 6 shows that the gradient is formed by a weighted product of the incident wavefield $\mathbf{x}$ and the adjoint wavefield $\mathbf{A}^{-1} \tilde{\mathcal{P}} \mathbf{S}_{d}^{\dagger} \mathbf{S}_{d} \mathbf{d}^{*}$. The gradient of the misfit function therefore requires computing only two forward problems per shot.

In practice, for realistically sized problems, resolving the Newton equation (equation 6) is avoided because of the large inherent cost. One alternative used in this study consists of considering only diagonal terms of the Hessian or the pseudo-Hessian matrix (Pratt et al., 1998; Shin et al., 2001) as a preconditioner for the optimization algorithm.

To overcome the diagonal estimation of the Hessian, an L-BFGS method can be used (Nocedal and Wright, 1999). This algorithm is more efficient than the preconditioned nonlinear conjugate gradient for solving FWI problems (Brossier et al., 2009). The algorithm estimates a nondiagonal inverse Hessian from the $m$ most recent gradient and model vectors. An initial estimate of the Hessian can be provided from the diagonal terms of an approximate Hessian. An example of the contribution of this method to improve the convergence level of the misfit function is illustrated later.

Once the right-hand side of equation 6 is estimated, the model is updated iteratively:

$$
\mathbf{m}^{l}=\mathbf{m}^{(l-1)}+\alpha \delta \mathbf{m}^{(l)},
$$

where $\alpha^{l-1}$ denotes the step length, estimated in this study by parabola fitting.

\section{Efficient mitigation of nonlinear effects}

FWI is carried out by proceeding iteratively from low to high frequencies. This allows short wavelengths to be introduced progressively in the parameter images and thus helps to mitigate the nonlinearity of the inverse problem. The strategy has proven effective for the acoustic inverse problem (Pratt, 1999; Ravaut et al., 2004; Operto et al., 2006). In the elastic case, work conducted to evaluate the ability of the method to locate small heterogeneities in shallow subsurface structures in the presence of a flat topography (Gélis, 2005) reports many difficulties stemming from the presence of complex wave phenomena, particularly surface waves. Because the waves contain most of the seismic energy and because they interact strongly with the topographic irregularities, we speculate that they will significantly govern the optimization process and constraint the algorithm to explore a wrong solution and reach a local minimum.

To fulfill our objective, i.e., imaging shallow and highly contrasted velocity structures in the presence of a complex topography, we must take into consideration three critical points.

1) An accurate starting model is required. It must be close enough to the true velocity model to avoid the cycle-skipping phenomenon, which may occur when the error traveltime is greater than half a period.

2) The receiver antenna must be long enough to ensure good model illumination. Limited-aperture acquisition geometries can result in the algorithm being trapped in a local minimum.
3) The choice of inverted frequencies is critical to guarantee 324 accurate coverage in terms of long and short wavelengths, 325 especially for the $\mathrm{S}$-wave velocity parameter $V_{\mathrm{S}}$. Low fre- 326 quencies must be considered to avoid convergence toward a 327 local minimum at an early stage. This restriction also explains 328 the necessity of considering a starting model close enough to 329 the real one. Moreover, selection of the inverted frequencies 330 must ensure a continuous wavenumber illumination follow- 331 ing, for example, the strategy proposed by Sirgue and Pratt 332 (2004).

An alternative to mitigate the strong nonlinearities resulting 335 from complex wave phenomena consists of defining two levels 336 of hierarchy (Brossier et al., 2009). The first is to perform suc- 337 cessive inversions of overlapping groups of finite frequencies to 338 better constrain the algorithm and take into account the redundant 339 information contained in the selected frequencies. Frequencies of 340 each group are inverted simultaneously, and the overlapping (fre- 341 quencies in common) between two successive groups is mini- 342 mized. Application of this strategy to the SEG/EAGE overthrust 343 model reveals some improvements in comparison to a sequential 344 single-frequency approach.

The second level consists of progressively introducing later 346 arrivals (converted waves, surface waves) in the inversion. In 347 the time domain, this level of hierarchy can be implemented 348 in a flexible way by time windowing (Pratt and Shipp, 1999). In 349 the frequency domain, only time damping can be used (Shin 350 et al., 2002). Time damping of a seismic signal $x(t)$ with respect 351 to the first-arrival traveltime $t_{0}$, for example, can be implemented 352 in the frequency domain by introducing a complex-valued fre- 353 quency following the expression

$X(w+i \gamma) \exp \left(\gamma t_{0}\right)=\int_{-\infty}^{+\infty} x(t) \exp \left(-\gamma\left(t-t_{0}\right)\right) \exp (-i w t) d t$,

where $\gamma$ denotes the applied damping factor.

To assess the effectiveness of these strategies in our context, 356 we conducted a numerical study for a realistic landslide model. 357 For all tests presented hereafter, the inverted model parameters 358 are $\mathrm{P}$ - and $\mathrm{S}$-wave velocities. The source-parameter estimate is 359 not addressed, although it is a critical issue when applying FWI 360 to real data. The proposed numerical tests are performed to 361 highlight two aspects. In the first section, we evaluate the per- 362 formance of the defined preconditioning strategies to recover the 363 velocity structures and to assess the contribution of the L-BFGS 364 optimization method. In the second section, we study the effect 365 of decimating the acquisition geometry on the inversion results, 366 notably in term of number of sources, to be as close as possible 367 to realistic cases.

\section{LANDSLIDE SYNTHETIC CASE STUDY: A NUMERICAL EXPERIMENT}

The landslide model was inspired from a transverse section of 371 the Super-Sauze earthflow located in the French Alps (Flageollet 372 et al., 2000). It consists of a $210 \times 60$-m section composed of 373 several velocity layers, as proposed by Grandjean et al. (2006), 374 after performing first-arrival tomography. The medium is 375 
characterized by strong lateral velocity variations associated with highly contrasted media, with $\mathrm{P}$ - and $\mathrm{S}$-wave velocities varying from 800 to $3200 \mathrm{~m} / \mathrm{s}$ and 480 to $1600 \mathrm{~m} / \mathrm{s}$, respectively (Figure 1; Table 1) with an inconstant Poisson's ratio. Here we used a constant density of $1600 \mathrm{~g} / \mathrm{cm}^{3}$ for forward and inverse problems. The surface topography is highly irregular.

Simulations were performed using a Ricker source wavelet with a peak frequency of $60 \mathrm{~Hz}$. In a real data context, the source signature and radiation pattern are additional unknowns that can be estimated by solving a linear inverse problem (Pratt, 1999; Virieux and Operto, 2009). The chosen parameters correspond to wavelengths (at the central frequency) varying between 53.3 and $13.4 \mathrm{~m}$ for $V_{\mathrm{P}}$ and between 26.6 and $8 \mathrm{~m}$ for $\mathrm{S}$-wave velocity $V_{\mathrm{S}}$. The frequency bandwidth covers the interval $[10,150] \mathrm{Hz}$. Detecting shallow structures of metric scale is thus affected by the bad resolution of the thin layers of the model. From a numerical point of view, a source with higher-frequency content should overcome this limitation. However, this assumption is meaningless in practice because high-frequency signals are strongly attenuated in the shallow, fissured layers of the medium and thus useless.

The mesh is divided into a 1-m-thick unstructured layer for P1 interpolation and a structured layer (made of equilateral triangles) for P0. This choice ensures at least 15 grid cells per minimum propagated wavelength (corresponding to the surface wave estimated from the shear wave and the Poisson's ratio with the Viktorov formula [Viktorov, 1965]) for the highest modeled frequency.

A total of 197 explosive (Ricker) sources were considered 1 $\mathrm{m}$ below the surface, with a $1-\mathrm{m}$ spacing along the horizontal axis; 197 receivers were located $0.5 \mathrm{~m}$ below the surface. Vertical and horizontal particle velocities were computed. An example of vertical and horizontal components of one shot gather (Figure 2) shows that most of the seismic energy is radiated in the form of surface waves. It also highlights the footprint of the irregular topography on the seismic signal, which is drastically warped. Strong diffractions resulting from the topography shape can be observed, particularly for the incident surface waves.

\section{Impact of inversion conditioning}

In our tests, $V_{\mathrm{P}}$ and $V_{\mathrm{S}}$ are the inverted model parameters, and density is supposed to be known. Starting models are smoothed versions of true ones, obtained after applying a 2D Gaussian smoothing function with a spatial correlation length of $6 \mathrm{~m}$ for $V_{\mathrm{S}}$ (Figure 3) and $V_{\mathrm{P}}$ parameters. This constitutes a

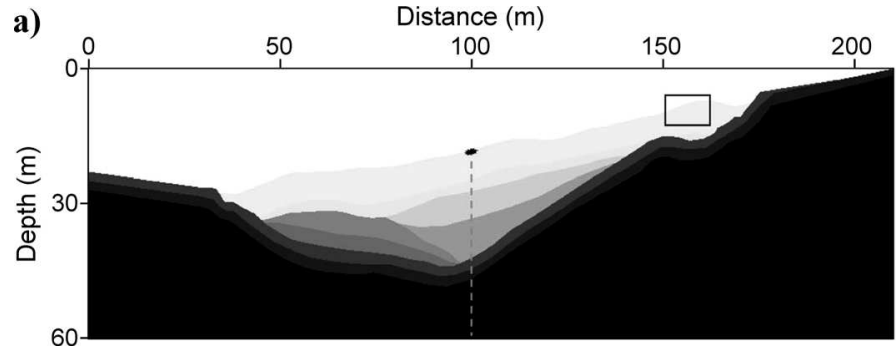

good compromise between severely altering the delineation of 420 layer interfaces and avoiding the cycle-skipping phenomenon 421 that may occur when the starting models are too far from the 422 real ones. For the shallow layers, it is a realistic model that can 423 be obtained by conventional methods. These models also sup- 424 pose that we have a priori knowledge on the shape of the bed- 425 rock. Examples of vertical and horizontal components of shot 426 gathers are shown in Figures $4 \mathrm{a}$ and $4 \mathrm{~b}$, for comparison with 427 those of Figures $2 \mathrm{a}$ and $2 \mathrm{~b}$.

\section{Successive inversions of single frequencies}

In a first step, sequential inversion is performed with respect 430 to the selected frequencies of Table 2 to ensure a continuous 431 wavenumber illumination (Sirgue and Pratt, 2004). Horizontal 432 and vertical components are considered. A maximum of 25 iter- 433 ations per frequency is performed to ensure convergence of the 434 algorithm at reasonable computing cost.

435

The final models obtained are shown in Figures 5a and 6. 436 The inversion fails to converge toward an acceptable model for 437 $V_{\mathrm{P}}$ and $V_{\mathrm{S}}$. Indeed, the main features of the layered structure are 438 not recovered. In addition, strong artifacts are observed. The 439 algorithm has converged into a local minimum because we 440 observe strong, unrealistic anomalies near the free surface 441 (Figure $5 \mathrm{~b}$ and Figure $7 \mathrm{~b}$ ). This failure can be attributed to the 442 dominant contribution of surface waves that prevents the high- 443 frequency signals associated with body waves to be considered 444 in the inversion. Similar effects have been observed by Gélis 445 et al. (2007) and Romdhane et al. (2009).

\section{Frequency group inversion of damped data}

In this section, we investigate the performance of a simultane- 448 ous inversion of damped data. We consider three overlapping 449 groups of three frequencies (see Table 2), with damping 450

Table 1. Maximum and minimum velocity parameters for the landslide model. Maximum and minimum wavelengths are calculated for the lowest and highest inverted frequencies, respectively.

\begin{tabular}{rrrrrc}
$\begin{array}{l}V_{\mathrm{P}} \\
(\mathrm{m} / \mathrm{s})\end{array}$ & $\begin{array}{l}V_{\mathrm{S}} \\
(\mathrm{m} / \mathrm{s})\end{array}$ & $\begin{array}{l}\lambda_{V_{\text {Pmax }}} \\
(\mathrm{m})\end{array}$ & $\begin{array}{l}\lambda_{V_{\text {Pmin }}} \\
(\mathrm{m})\end{array}$ & $\begin{array}{l}\lambda_{V_{\text {Smax }}} \\
(\mathrm{m})\end{array}$ & $\begin{array}{c}\lambda_{V_{\text {Smin }}} \\
(\mathrm{m})\end{array}$ \\
\hline 800 & 480 & 23.8 & 5.9 & 11.9 & 3.57 \\
3200 & 1600 & 150.2 & 37.6 & 75.1 & 22.5 \\
\hline
\end{tabular}

b)
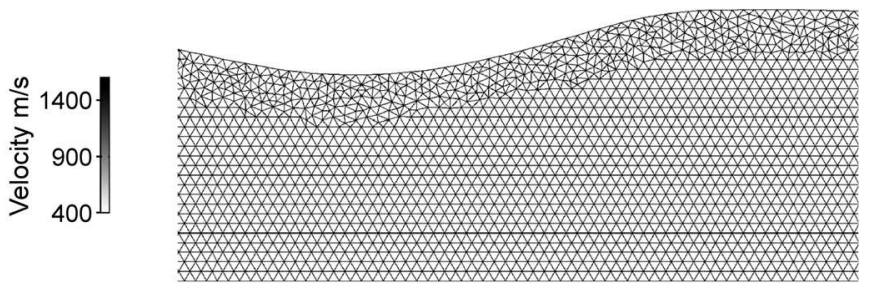

Figure 1. (a) $V_{\mathrm{S}}$ true velocity model for the (realistic) landslide model. The gray dashed line and the black star correspond to the position of the extracted vertical profiles and the source position of shot gathers depicted in the following figures, respectively. (b) Zoom of the boxed area, showing the mesh used for the landslide model with the mixed DG P0-P1 method. 


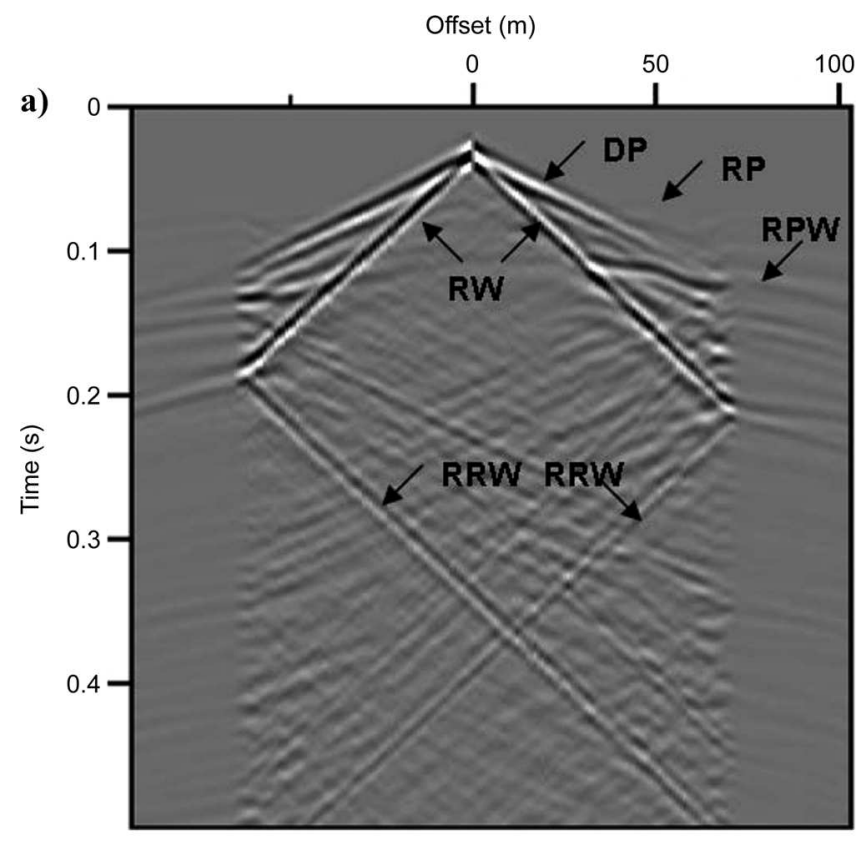

b)



Figure 2. Examples of (a) horizontal and (b) vertical synthetic shot gathers of the landslide model. The shot position correspond to an abscissa of $100 \mathrm{~m}$ on the horizontal distance axis of Figure 1. DP, RP, and RPW correspond to direct, refracted, and reflected $\mathrm{P}$-waves, respectively. RW and RRW correspond to Rayleigh waves (fundamental mode) and back-propagated Rayleigh waves.
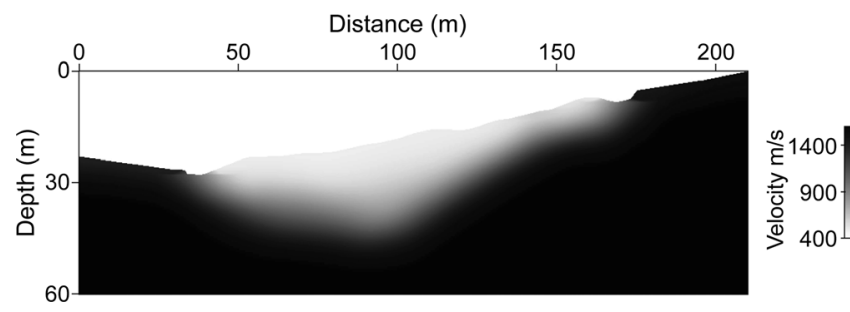

Figure 3. Starting $V_{\mathrm{S}}$ model considered for the landslide case. coefficients varying between 20 and 1.5. Figures $4 \mathrm{c}$ and $4 \mathrm{~d} 451$ shows the vertical and horizontal components obtained with a 452 damping coefficient of 20. The shot position used in Figure 2 is 453 considered. Comparison of shot gathers of Figures 2 and 4 high- 454 lights the role of data damping to progressively introduce 455 surface waves as well as complex free-surface reflections, par- 456 ticularly for the far offsets. A maximum of 25 iterations was 457 performed for each damped frequency group.

458

Final results, obtained after inverting the three groups (Fig- 459 ures 8 and 9), reveal how crucial this strategy is to converge 460 successfully toward an acceptable solution. For $V_{\mathrm{S}}$, focusing the 461 shallower structures is defined with a high level of resolution. 462 For the deeper layers, the model is slightly improved and the 463 contribution is less significant as the velocity values (and thus 464
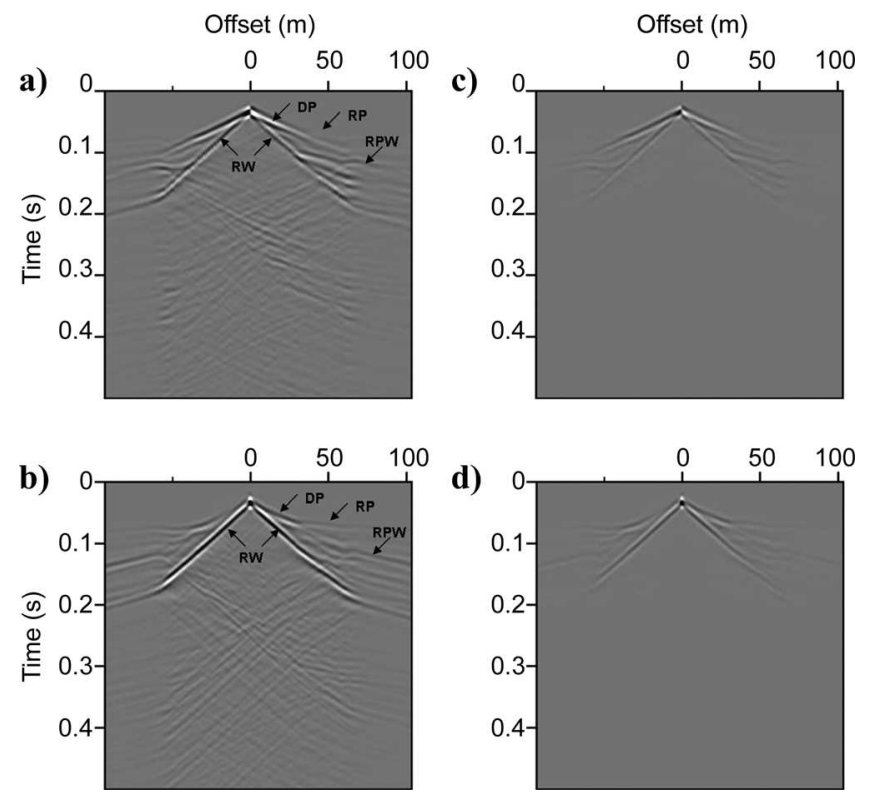

Figure 4. (a) Horizontal and (b) vertical synthetic shot gathers of the starting landslide model. (c) Damped horizontal and (d) vertical synthetic shot gathers of the starting landslide model $(\gamma=20)$. The shot position correspond to an abscissa of $100 \mathrm{~m}$ on the horizontal distance axis of Figure 1. DP, RP, and RPW correspond to direct, refracted, and reflected P-waves, respectively. RW correspond to Rayleigh waves (fundamental mode).

Table 2. Sequential inverted frequencies, frequency groups, and damping coefficients considered for the landslide model.

\begin{tabular}{lccc}
\hline $\begin{array}{l}\text { Frequency } \\
\text { group }\end{array}$ & $\begin{array}{c}\text { Sequential } \\
\text { frequency } \\
(\mathrm{Hz})\end{array}$ & $\begin{array}{c}\text { Simultaneous } \\
\text { frequency } \\
(\mathrm{Hz})\end{array}$ & $\begin{array}{c}\text { Damping } \\
\text { coefficient } \\
(1 / \mathrm{s})\end{array}$ \\
\hline 1 & 21.3 & $21.3,27.5,42.7$ & $20,5,1.51$ \\
2 & 27.5 & $42.7,61.0,82.4$ & \\
3 & 42.5 & $82.4,106.8,134.3$ & \\
4 & 61.0 & & \\
5 & 82.4 & & \\
6 & 106.8 & & \\
7 & 134.3 & & \\
\hline
\end{tabular}


the associated wavelengths) increase (Figures 8 and 10b). The weak contribution of the inversion process to reconstruct $V_{\mathrm{P}}$ (Figures 9 and 10a) was expected and can be explained by the lack of short wavelengths illuminated with respect to the $V_{\mathrm{P}}$ model velocities (see the wavelengths associated with $V_{\mathrm{P}}$ in Table 2). Few artifacts can, however, be noticed in the zones corresponding to the highest velocity contrasts (between the shallowest layer and the bedrock, in Figure 8b).

Examples of an initial differential seismogram (difference between data calculated with the true model and data calculated with the starting model) and a final differential seismogram (difference between data calculated in the final model and data calculated with the true model) are depicted in Figures 10c and 10d, respectively. The comparison shows that the unexplained energy mainly comes from the back-propagated Rayleigh waves at the highest velocity contrasts.

The penetration depth of the Rayleigh wave is approximately half of its wavelength. This means it will dominate the low-frequency part of the data spectrum, whereas body waves will dominate the high-frequency part of the spectrum. Applying strong damping coefficients to the high frequencies to favor the use of body waves is therefore unnecessary, in our opinion.

We have also investigated the effect of resampling the frequency interval in the inversion group. We have divided the frequency interval by a factor of two and considered three groups of five (instead of three) frequencies. This resampling is

a)
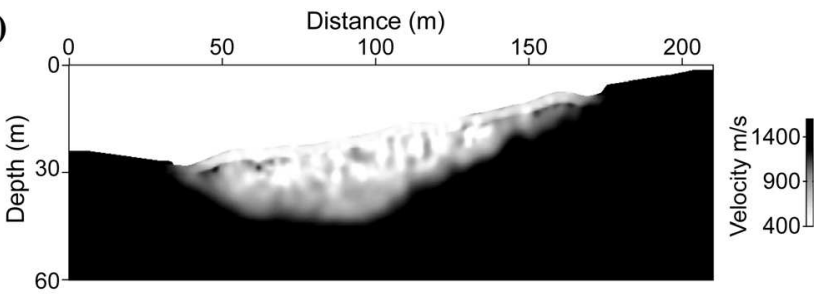

b)


Figure 5. (a) Final $V_{\mathrm{S}}$ model and (b) relative velocity error (ratio of the velocity error to the true velocity), obtained after sequential inversion of seven frequencies varying from $21.3-134.3 \mathrm{~Hz}$ as indicated in Table 2. Vertical and horizontal components are inverted.

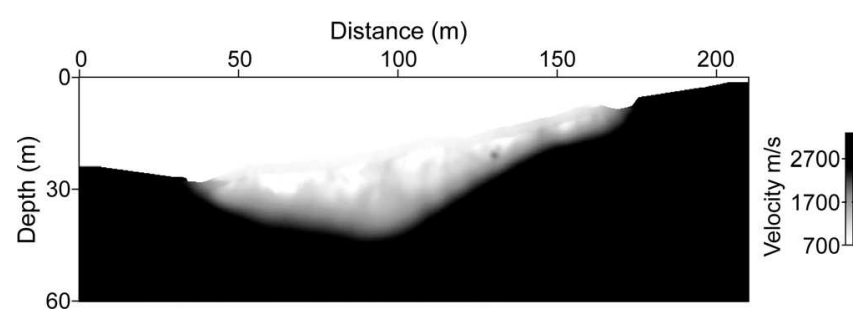

Figure 6. Final $V_{\mathrm{P}}$ model obtained after sequential inversion of seven frequencies varying from 21.3 to $134.3 \mathrm{~Hz}$ as indicated in Table 2. Vertical and horizontal components are inverted. expected to strengthen the spectral redundancy and yield a 491 higher definition of layers. The same damping coefficients were 492 considered as in the previous example. Results in Figure 11493 show a slight improvement of $V_{\mathrm{S}}$ parameter reconstruction at 494 the expense of a significantly higher computing cost.

\section{Contribution of L-BFGS method}

496

We performed an inversion test using the L-BFGS optimiza- 497 tion method with the same frequency groups and damping coef- 498 ficients as in the previous section (with respect to Table 2). The 499 initial estimate of the Hessian is provided by the diagonal ele- 500 ments of the pseudo-Hessian (Shin et al., 2001), and five differ- 501 ences of cost-function gradients and model vectors are used for 502 the L-BFGS algorithm. Figures 12a and 12b and Figures 13a 503 and 13b show final $V_{\mathrm{P}}$ and $V_{\mathrm{S}}$ reconstructed velocity models and 504 vertical extracted profiles for each parameter, respectively. 505
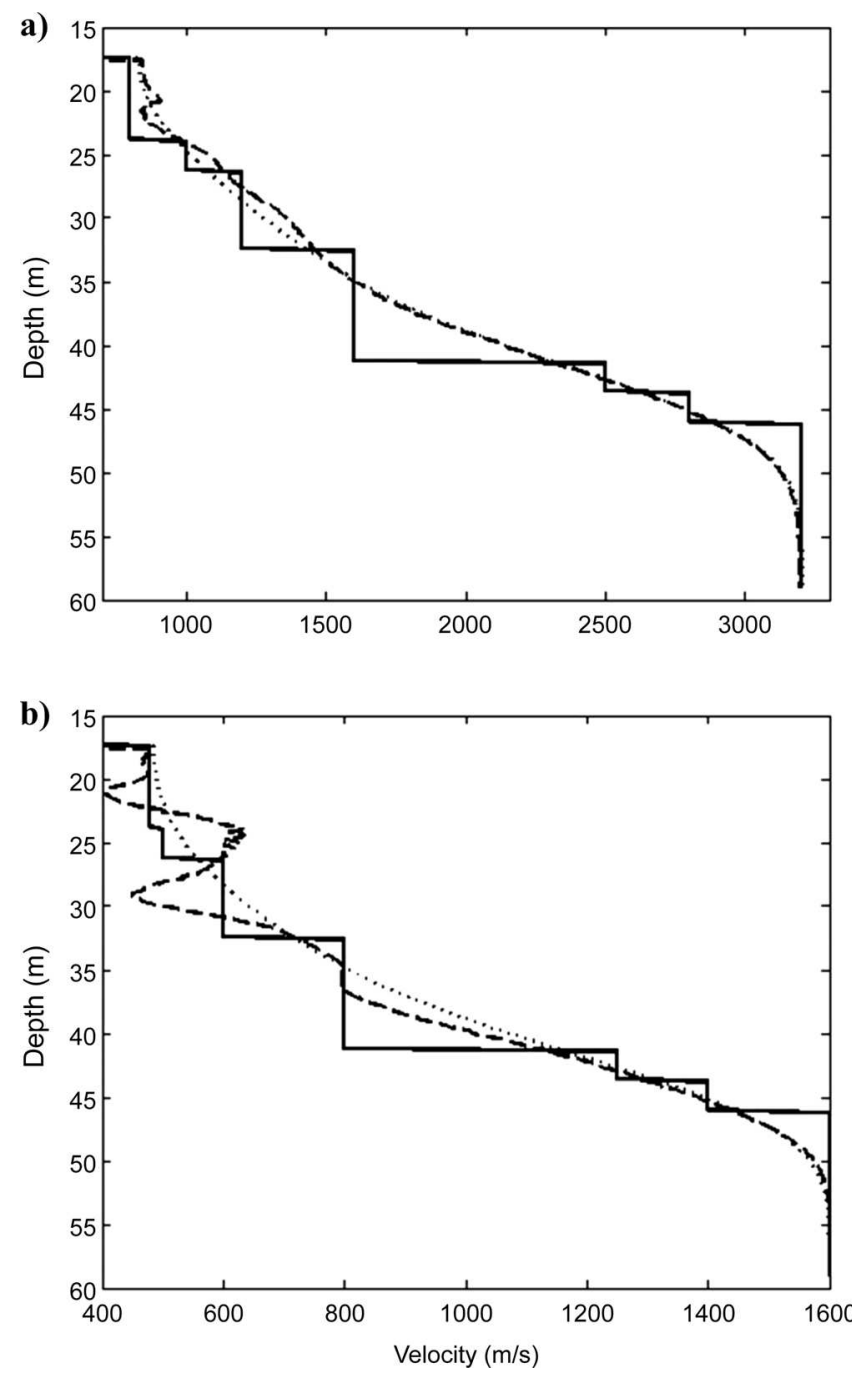

Figure 7. (a) $V_{\mathrm{P}}$ and (b) $V_{\mathrm{S}}$ parameter profiles extracted along a vertical line (located at a distance of $100 \mathrm{~m}$ ) obtained after sequential inversion of seven frequencies varying from 21.3 to $134.3 \mathrm{~Hz}$ as indicated in Table 2. Profiles of the true model are plotted with solid black lines, the initial model is the dotted lines, and the final model is the dashed lines. 

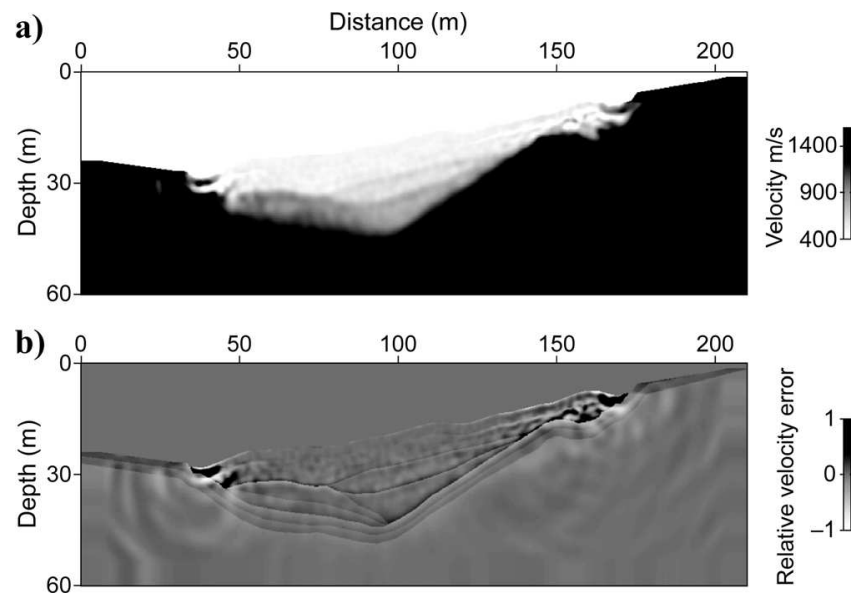

Figure 8. (a) Final $V_{\mathrm{S}}$ model and (b) relative velocity error (ratio of the velocity error to the true velocity), obtained after simultaneous inversion of three damped-frequency groups, varying from 21.3 to $134.3 \mathrm{~Hz}$, as indicated in Table 2. Vertical and horizontal components are inverted. Significant velocity errors can be observed near the free surface.

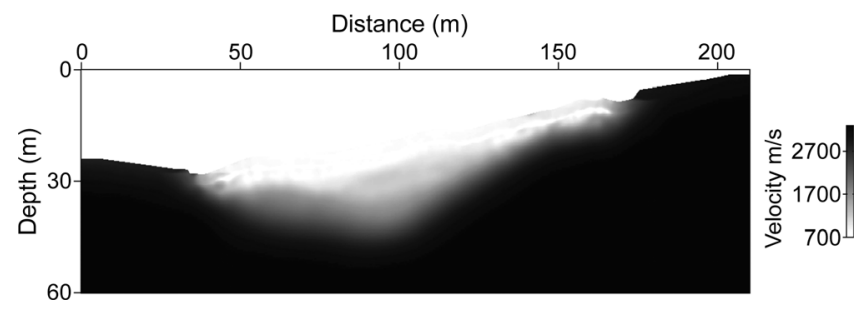

Figure 9. Final $V_{\mathrm{P}}$ model obtained after simultaneous inversion of three damped-frequency groups varying from 21.3 to $134.3 \mathrm{~Hz}$ as indicated in Table 2. Vertical and horizontal components are inverted.

Figure 10. (a) $V_{\mathrm{P}}$ and (b) $V_{\mathrm{S}}$ parameter profiles extracted along a vertical line (located at a distance of $100 \mathrm{~m}$ ) obtained after simultaneous inversion of three damped-frequency groups, varying from 21.3 to $134.3 \mathrm{~Hz}$, as indicated in Table 2. True model is plotted in solid black lines, initial model in dotted lines, and final model in dashed lines. (c) Vertical component of an initial differential seismic section (difference between data calculated with the true model and data calculated with the starting model). (d) Vertical component of a final differential seismogram (difference between data calculated in the final model and data calculated with the true model).

(a)

Amplitudes of the structures are significantly better defined for 506 the shallow and the deep layers of the model in comparison 507 with those obtained with the PCG optimization method (see Fig- 508 ures $8 \mathrm{a}, 9,10 \mathrm{a}$, and 10b). Fewer artifacts can be observed even 509 in the shallow zone corresponding to the highest velocity 510 contrasts.

Figure12c depicts the evolution of the logarithm of the misfit 512 function with respect to the iteration number with the L-BFGS 513 and PCG methods for the first frequency group. The conver- 514 gence level is drastically improved with the L-BFGS algorithm 515 when compared to PGC. The final differential seismograms 516 (Figure 14) show that amplitude residuals are strongly attenu- 517 ated, even for the longer recording times. The strong residuals 518 (observed in Figure 10d) from the highest velocity contrasts are 519 significantly lessened. Similar effects have been noticed by 520 Brossier et al. (2009) and attributed to the contribution of the 521 off-diagonal terms of the Hessian matrix estimated by the L- 522 BFGS algorithm. This confirms the promising prospects for real- 523 istic applications.

\section{Impact of data decimation}

In this section, we analyze the impact of data decimation on 526 the inversion results. The percentage of model degradation with 527 respect to a reference case is estimated with the root mean 528 square (rms) of the relative velocity error.

\section{Component selection}

In practice, seismic investigations for shallow-structure char- 531 acterizations are usually restricted to recording the vertical parti- 532 cle-velocity component. This limitation represents an additional 533 ambiguity for the inverse problem.

Two configurations were tested by considering the vertical or 535 horizontal component of the synthetic data. The same frequency 536
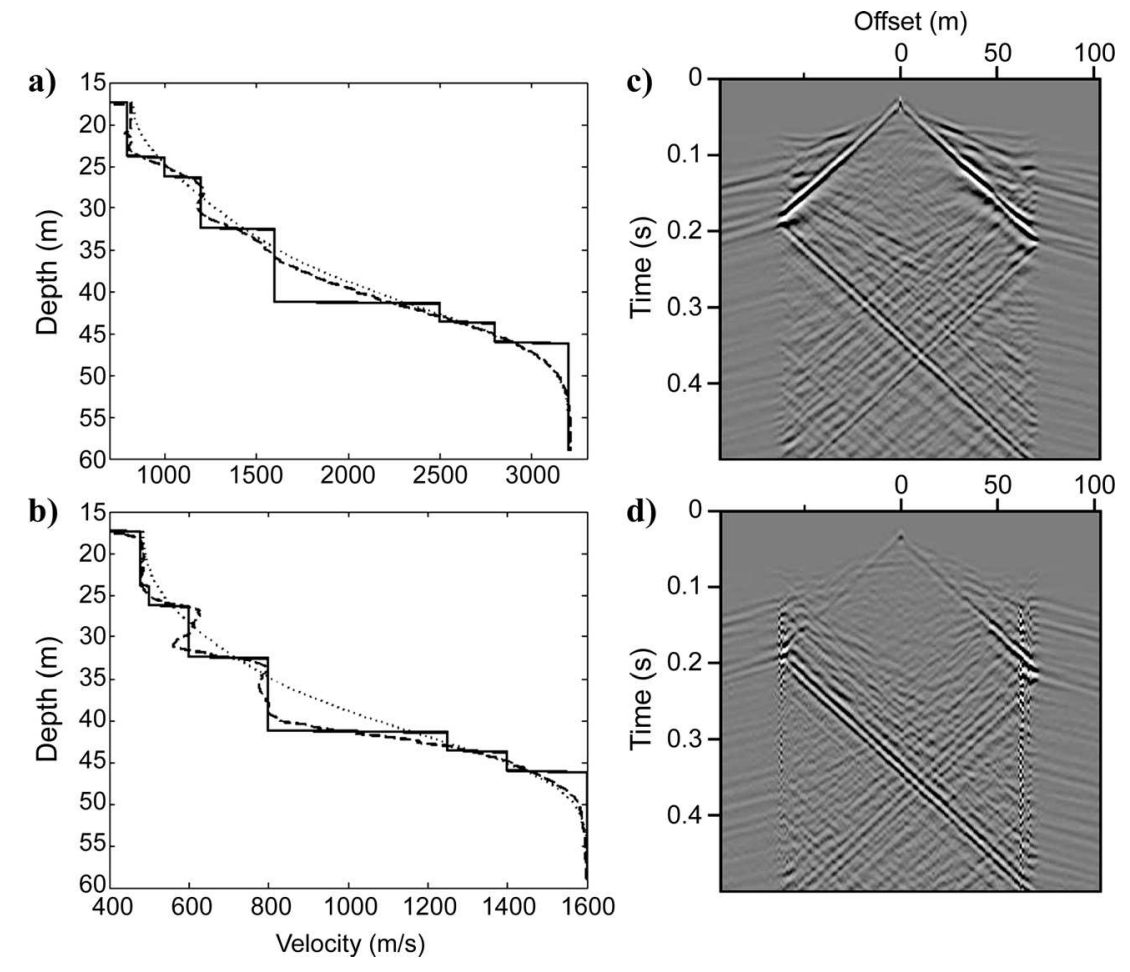
groups and damping terms were used as in the previous section for consistency. The final distribution of $V_{\mathrm{S}}$ reconstructed by considering the vertical component has a higher resolution (Figures $15 \mathrm{a}$ and $15 \mathrm{c}$ ) compared to the one obtained by considering
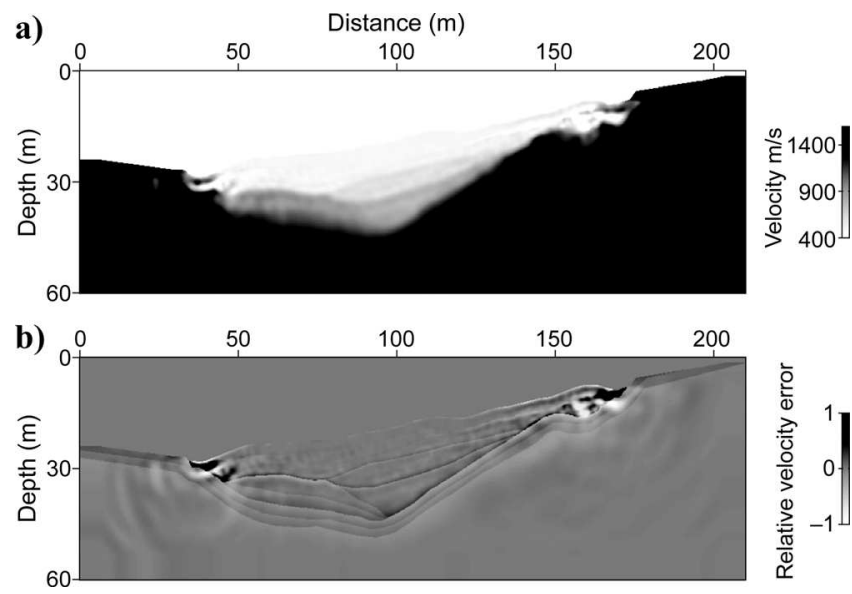

Figure 11. (a) Final $V_{\mathrm{S}}$ model and (b) relative velocity error (ratio of the velocity error to the true velocity), obtained after simultaneous inversion of three damped-frequency groups varying from 21.3 to $134.3 \mathrm{~Hz}$. Five frequencies per group are used in the inversion. Horizontal and vertical components are considered.
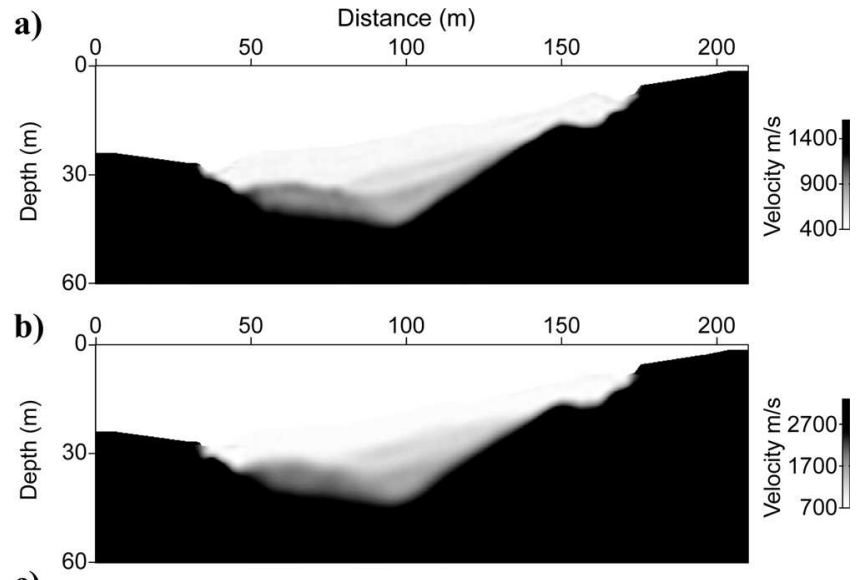

c)

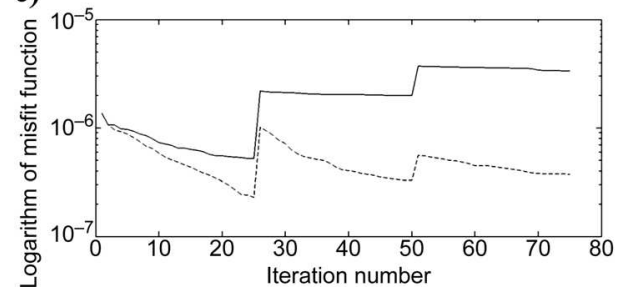

Figure 12. Final (a) $V_{\mathrm{S}}$ and (b) $V_{\mathrm{P}}$ models obtained after simultaneous inversion of three damped-frequency groups varying from 21.3 to $134.3 \mathrm{~Hz}$ with the L-BFGS algorithm. Three frequencies per group are used in the inversion. Horizontal and vertical components are considered. (c) Evolution of the L-BFGS and the PCG logarithm of the objective function with respect to the iteration number for the inversion of the first frequency group with three damping coefficients (see Table 2). Twenty-five iterations are performed for each damping coefficients. The L-BFGS algorithm drastically improves the convergence level of the objective function. the horizontal component. In the better case, we observe large 541 velocity errors close to the free surface in the zones with a very 542 high contrast between the steeply dipping bedrock and the shal- 543 lowest layer (Figures $15 \mathrm{~b}$ and 15d). Percentages for $V_{\mathrm{S}}$ model 544 degradation (with respect to the reference case of Figure $8 \mathrm{~b}$ ) are 545 $24 \%$ with the vertical component and $102 \%$ with the horizontal 546 component. As a result, the algorithm appears more sensitive to 547 the information provided by the vertical data component. 548

In addition, final inverted $V_{\mathrm{S}}$ images obtained by considering 549 the vertical component do not differ significantly from the ones 550 calculated by considering vertical and horizontal components. 551 However, a comparison of the figures of associated relative ve- 552 locity errors (Figures $8 \mathrm{~b}$ and $15 \mathrm{~b}$ ) shows the lower resolution of 553 the deeper layers of the model.

\section{Acquisition configuration}

We finally investigate the sensitivity of inversion results to 556 theparameters of the recording geometry. The impact of 557
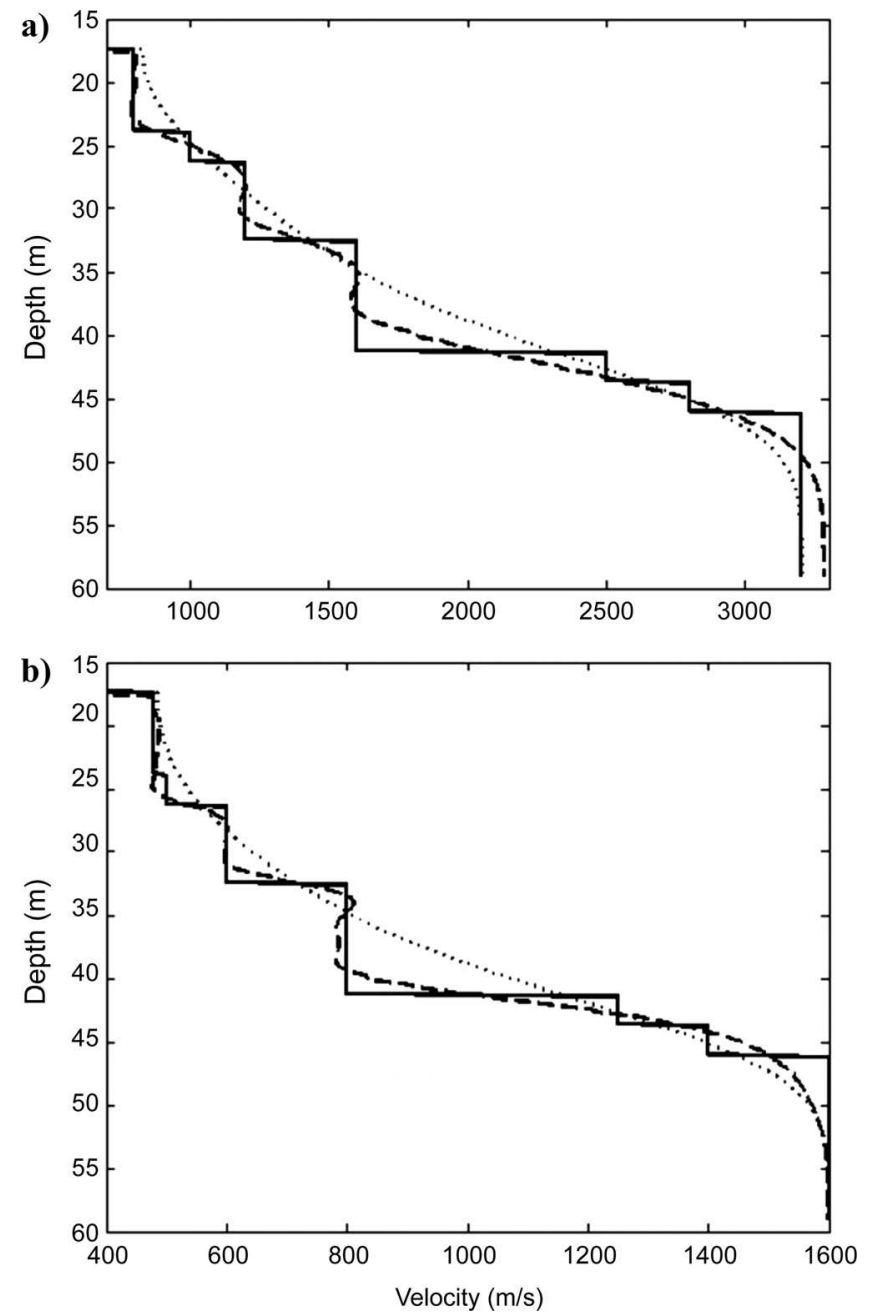

Figure 13. (a) $V_{\mathrm{P}}$ and (b) $V_{\mathrm{S}}$ parameter profiles extracted along a vertical line (located at a distance of $100 \mathrm{~m}$ ) obtained after simultaneous inversion of three damped-frequency groups varying from 21.3 to $134.3 \mathrm{~Hz}$ as indicated in Table 2 . The true model is plotted in solid black lines, the initial model is a dotted line, and the final model is a dashed line. 
decimating survey geometries on the waveform tomography for lithospheric imaging is addressed by Brenders and Pratt (2007). The requirement of fully unaliased surface sampling $\Delta_{\text {samp }}$ is given by the relationship $(\Delta r, \Delta s) \leq \Delta_{\text {samp }}=\lambda / 2$, where $\Delta r$ and $\Delta s$ denote receiver and source spacings and $\lambda$ is the calculated wavelength for a specific frequency with respect to the minimum velocity of the medium. Brenders and Pratt (2007) suggest that for a receiver spacing below $\lambda / 2$, the image quality remains acceptable for $\Delta s \simeq 3 \Delta_{\text {samp. }}$. In the context of near-surface imaging, field and logistic limitations often prevent the use of a

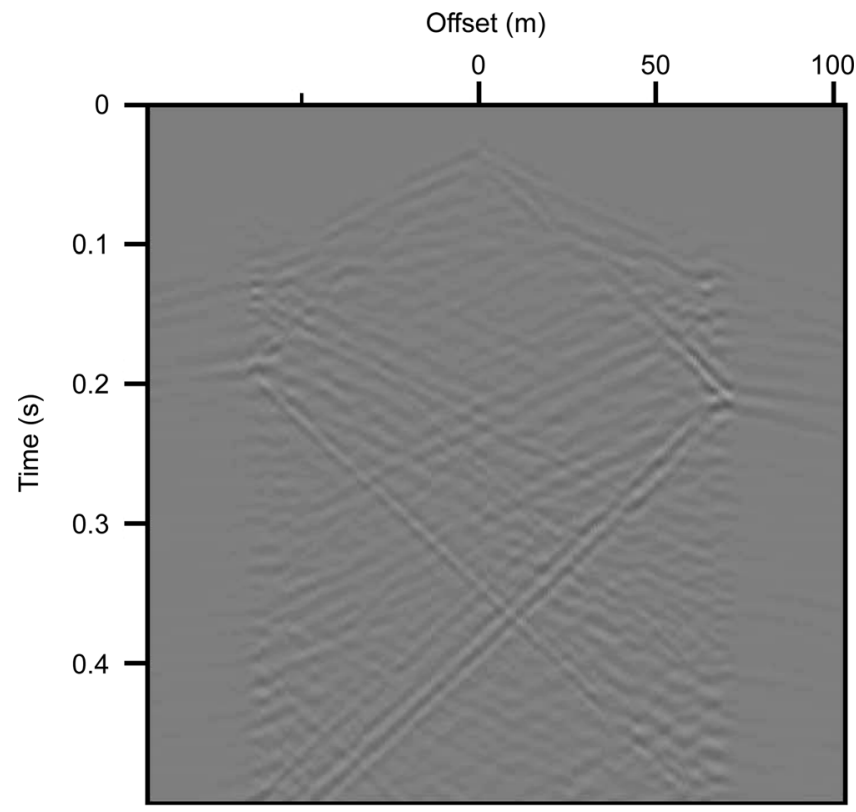

Figure 14. Vertical component of a final differential seismic section (difference between data calculated in the final model and data calculated in the true model) obtained after running the inversion with the L-BFGS algorithm. Amplitude residuals are strongly attenuated, compared with those obtained with the PCG algorithm (Figure 10d).
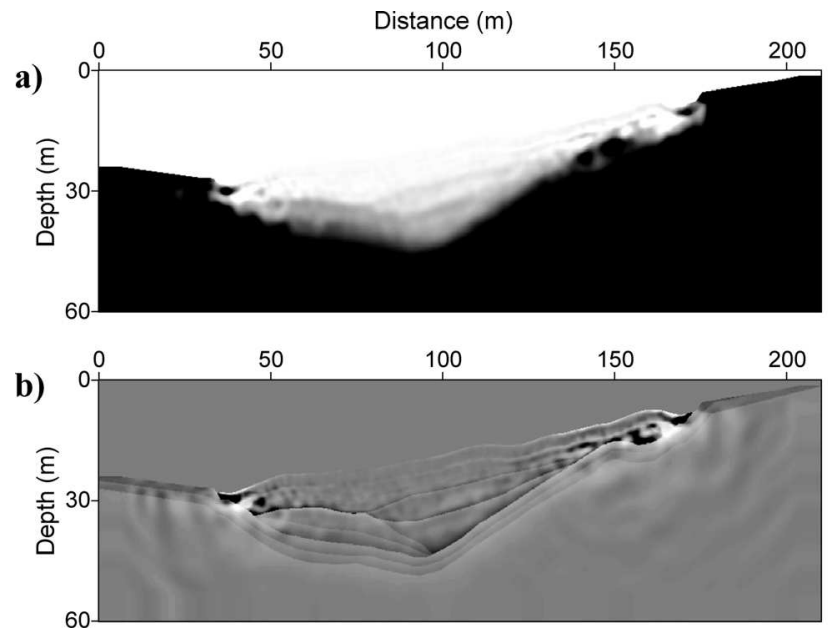

dense sampling of source array. We keep the number of receiver 568 constant and evaluate the effect of decimating the number of 569 sources with a sparser grid consisting of 99 sources (with a 2-m 570 spacing) and then 49 sources (with a 4-m spacing), respectively. 571 Recalling that the minimum wavelength with respect to the S- 572 wave velocity is computed from $V_{\mathrm{S}_{\min }}=480 \mathrm{~m} / \mathrm{s}$ and 573 $f_{\max }=134.2 \mathrm{~Hz}$, we have $\Delta r=1 m \leq \Delta_{\text {samp }}=1.78 \mathrm{~m}$ and 574 $\Delta s<3 \Delta_{\text {samp }} \simeq 3 \lambda_{\min } / 2 \simeq 5.34 \mathrm{~m}$ for both cases. The minimum 575 and maximum offset coverages along the model are retained. 576 Only the vertical data component is considered for the 577 inversion.

Although the acquisition aperture is not modified, this acqui- 579 sition geometry is expected to mitigate the inversion perform- 580 ance slightly. Results show that a source sampling of $2 \mathrm{~m}$ leads 581 to acceptable results (Figures 16a and 16b) in comparison to 582 those obtained with a $1-\mathrm{m}$ source sampling. The percentage of 583 model degradation is $12 \%$. However, associated vertical profiles 584 depicted in Figures $17 \mathrm{a}$ and $17 \mathrm{~b}$ demonstrate that the deep struc- 585 ture (with a $V_{\mathrm{S}}$ velocity of $800 \mathrm{~m} / \mathrm{s}$ ) is defined with a lower def- 586 inition. A source sampling of $4 \mathrm{~m}$ introduces significant aliasing 587 effects near the free surface (Figures $16 \mathrm{c}$ and 16d), translated 588 into strong artifacts. The inversion obviously fails to converge 589 toward the true model with an acceptable level of resolution. 590 The final $V_{\mathrm{S}}$ image obtained is severely altered, with respect to 591 Figure 15a. The percentage of model degradation is $43 \%$. 592

Extracted vertical profiles (Figures 17c and 17d) confirm the 593 aliasing effect observed near the free surface and the poor reso- 594 lution of the deep structures. This effect can be even more pro- 595 nounced in the presence of noise in real data, which seriously 596 mitigates the effectiveness of the algorithm to improve layer 597 definition.

\section{Computing time}

We used a constant mixed P0-P1 mesh with a total of 600 266,709 cells composed of 8232 P1 cells (with three degrees 601 of freedom per field) and 258,477 P0 cells (with one degree of 602 freedom per field), giving 1,415,865 degrees of freedom. The 603 forward modeling required an average time of $89 \mathrm{~s}$ to be solved 604

Figure 15. (a) Final $V_{\mathrm{S}}$ models and (b) relative velocity errors (ratio of velocity error to true velocity), obtained after simultaneous inversion of three damped-frequency groups varying from 21.3 to $134.3 \mathrm{~Hz}$, as indicated in Table 2. Only the vertical (a, b) or horizontal (c, d) component data are inverted. 
for 197 sources per modeled frequency. For the inversion, each iteration required an average time of $400 \mathrm{~s}$ and a total memory of $6.7 \mathrm{~Gb}$ for factorization. All simulations were performed using 24 cores on a BRGM HP DL 165 G2 cluster, which consists of 32 nodes with Myrinet interconnection. Each node comprises two quad-core $2.3-\mathrm{GHz}$ AMD Opteron processors, providing $16 \mathrm{~Gb}$ of RAM.

\section{DISCUSSION}

We have applied elastic FWI inversion to a realistic landslide model characterized by strong lateral velocity variations and a complex surface topography. In this particular context, the seismic signal is dominated by surface waves that cannot be separated easily from body waves because of the limited aperture of the acquisition geometry. The inversion of raw data failed to 618 yield acceptable velocity images. This failure can be attributed 619 to the dominant contribution of surface waves. The effects of 620 surface waves on FWI have been investigated in small-scale 621 field experiments by Gélis et al. (2007) for the elastic case and 622 by Bleibinhaus and Rondenay (2009) in the presence of a com- 623 plex topography for the acoustic case on a larger scale. 624

The performed tests reveal that a combination of inversion of 625 overlapping groups of multiple frequencies and data damping to 626 allow a progressive introduction of the complex seismic events 627 (surface waves, multiples) is critical to mitigate the strong nonli- 628 nearities introduced by surface waves and to reconstruct the 629 shallow structures accurately. The use of a quasi-Newton L- 630 BFGS optimization algorithm can significantly improve the con- 631 vergence level of the method and the parameters reconstruction. 632
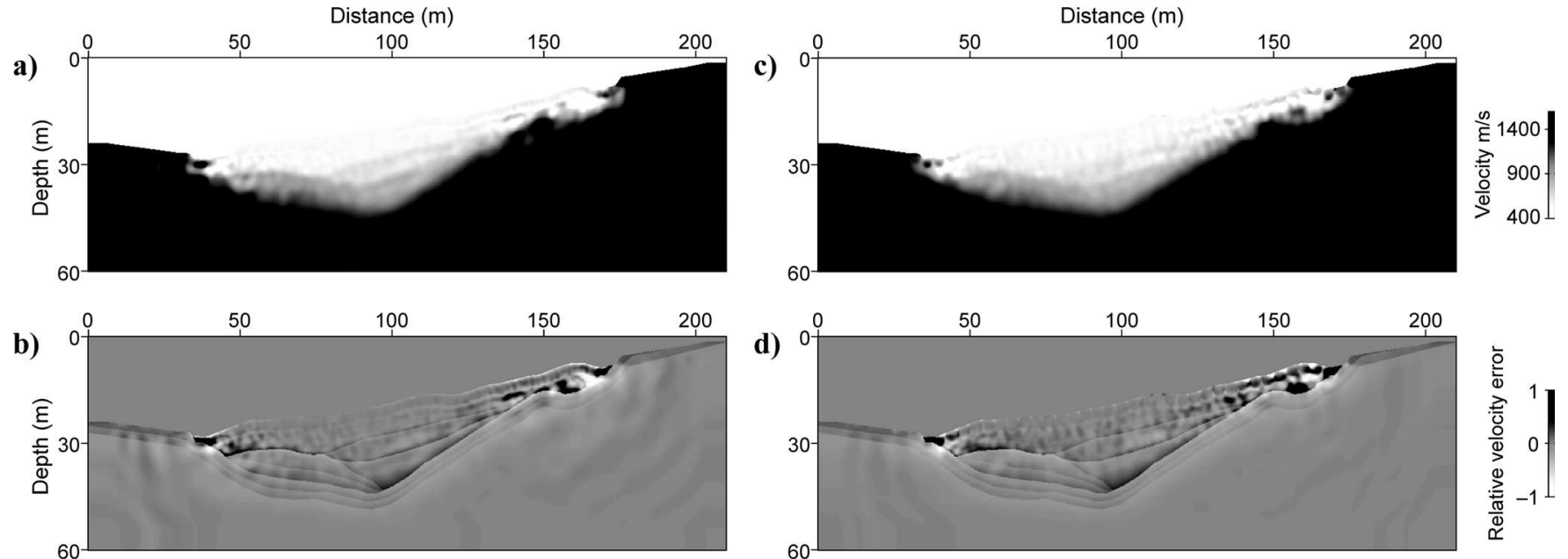

Figure 16. (a) Final $V_{\mathrm{S}}$ model and (b) relative velocity error (ratio of velocity error to true velocity), obtained after simultaneous inversion of three damped-frequency groups varying from 21.3 to $134.3 \mathrm{~Hz}$ as indicated in Table 2 . Only the vertical component is inverted and a decimated acquisition $(\Delta s=2 \mathrm{~m})$ is used. (c, d) Final $V_{\mathrm{S}}$ model and relative velocity error with $\Delta s=4 \mathrm{~m}$.
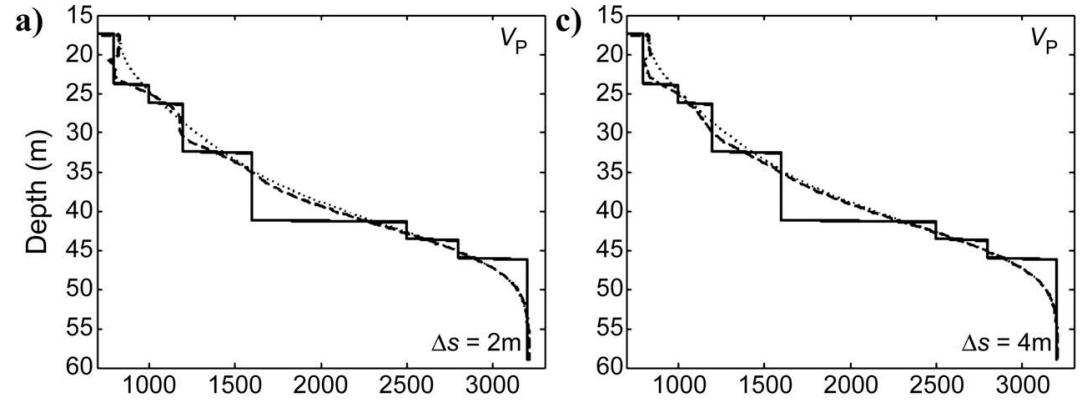

Figure 17. (a, c) $V_{\mathrm{P}}$ and (b, d) $V_{\mathrm{S}}$ parameter profiles extracted along a vertical line (located at a distance of $100 \mathrm{~m}$ ), obtained after simultaneous inversion of three damped-frequency groups varying from 21.3 to $134.3 \mathrm{~Hz}$, as indicated in Table 2. Only the vertical component is inverted. Two levels of decimation are considered with a source sampling of $2 \mathrm{~m}(\mathrm{a}, \mathrm{b})$ and $4 \mathrm{~m}(\mathrm{c}, \mathrm{d})$ along the surface topography. True model is plotted in solid black lines, initial model is plotted in dotted lines, and final model is plotted in dashed lines.
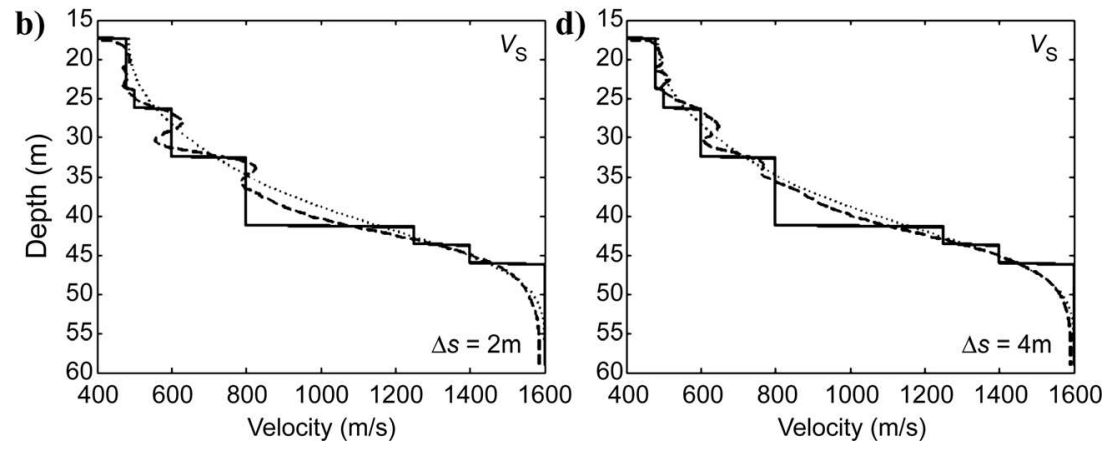
Efficiency of the process can, however, be severely altered by an insufficient source sampling interval.

An important extension to the elastic FWI, which may be critical for challenging real data applications, should incorporate the reconstructed attenuation parameters $Q_{\mathrm{P}}$ and $Q_{\mathrm{S}}$. Implementation of the algorithm in the frequency domain can take advantage of the complex-velocity Kolsky-Futterman model (Toverud and Ursin, 2005). Application to real data, in the context of near-surface characterization, can be based on the waveform-tomography workflow proposed by Smithyman et al. (2009) to produce images of $V_{\mathrm{P}}$ and $Q_{\mathrm{P}}$ parameters and to locate shallow targets. It also requires an estimation of the source (Pratt, 1999), which was supposed known in our work.

\section{CONCLUSION}

We have presented a numerical study to evaluate the potential of a 2D FWI approach; it shows promising prospects for imaging shallow structures in the presence of a complex topography. A discontinuous Galerkin method, based on a low-order mixed P0-P1 interpolation, is used for accurate wavefield modeling at a reasonable computing cost. A $2 \mathrm{D}$ elastic frequency-domain FWI algorithm has been applied to a realistic landslide model, characterized by highly contrasted layers and strong lateral velocity variations.

A two-level preconditioning strategy, based on simultaneous multifrequency inversion of damped data, has been applied to mitigate difficulties inherent in classical single-frequency inversions. Results confirm that simultaneous inversion of damped data, which allows a progressive introduction of converted and free surface waves, significantly outperforms the successive single-frequency inversion approach. It is a useful solution to mitigate strong nonlinearities resulting from surface waves and to avoid convergence toward a local minimum. We have also emphasized the high potential of the L-BFGS optimization method to improve the convergence level significantly, compared to the more classical PCG algorithm.

Finally, we have addressed the impact of some recording parameters on medium reconstruction. We have shown that restricting the inversion to the vertical component data can lead to acceptable results in terms of imaging resolution and convergence level, with a percentage of model degradation of $24 \%$. We have also illustrated how poor model illumination is translated in terms of imaging resolution. Future work will tackle the construction of the initial model, a key issue for FWI before considering applications to real data.

\section{ACKNOWLEDGMENTS}

The study was funded by BRGM and by Agence Nationale de la Recherche (ANR), project ANR-05-NT05-2-42427. The MUMPS software package was used for linear system solving and Triangle software was used for mesh generation. We are grateful to M. Dietrich and four anonymous reviewers for their comments and suggestions on this paper.

Bleibinhaus, F., and S. Rondenay, 2009, Effects of surface scattering in full-waveform in-version: Geophysics, 74, no. 6, WCC69-WCC77, doi:10.1190/1.3223315.

Bodet, L., 2005, Limites théoriques et expérimentales de l'inversion de la dispersion des ondes de Rayleigh: Apport de la modélisation numérique et physique: Ph.D. dissertation, Bureau de recherches geologiques et 690 minières/Laboratoire Central des Sponts et Chaussées (BRGM/LCPC). 691

Bohlen, T., and E. H. Saenger, 2006, Accuracy of heterogeneous 692 staggered-grid finite-difference modeling of Rayleigh waves: Geophysics, 693 71, no. 4, T109-T115, doi:10.1190/1.2213051. 694

Brenders, A. J., and R. G. Pratt, 2007, Full waveform tomography for 695 lithospheric imaging: Results from a blind test in a realistic crustal 696 model: Geophysical Journal International, 168, no. 1, 133-151, 697 doi:10.1111/j.1365-246X.2006.03156.x.

Brossier, R., 2009, Imagerie sismique à deux dimensions des milieux 699 visco-élastiques par inversion des formes d'onde: Dévelopements méth- 700 odologiques et applications: Ph.D. dissertation, Université de Nice- 701 Sophia-Antipolis.

_, 2010, Two-dimensional frequency-domain visco-elastic full 703 waveform inversion: Parallel algorithms, optimization and performance: 704 Computers \& Geosciences, doi:10.1016/j.cageo.2010.09.013. 705

Brossier, R., S. Operto, and J. Virieux, 2009, Seismic imaging of complex 706 onshore structures by 2D elastic frequency-domain full-waveform 707 inversion: Geophysics, 74, no. 6, WCC105-WCC118, doi:10.1190/ 708 1.3215771 .

Brossier, R., J. Virieux, and S. Operto, 2008, Parsimonious finite-volume 710 frequency-domain method for 2D $P-S V$-wave modelling: Geophysical 711 Journal International, 175, no. 2, 541-559, doi:10.1111/j.1365-246X. 712 2008.03839.x.

Červený, V., 2001, Seismic ray theory: Cambridge University Press. 714

Črvený, V., I. A. Molotkov, and I. Pšenčik, 1977, Ray theory in seismol- 715 ogy: Charles University Press.

Dumbser, M., and M. Käser, 2006, An arbitrary high-order discontinuous 717 Galerkin method for elastic waves on unstructured meshes - - II. The 718 three-dimensional isotropic case: Geophysical Journal International, 719 167, no. 1, 319-336, doi:10.1111/j.1365-246X.2006.03120.x.

Ellefsen, K. J., 2009, A comparison of phase inversion and traveltime to- 721 mography for processing near-surface refraction traveltimes: Geophy- 722 sics, 74, no. 6, WCB11-WCB24, doi:10.1190/1.3196857.

Flageollet, J. C., J. P. Malet, and O. Maquaire, 2000, The 3-D structure of 724 the Super-Sauze earthflow: A first stage toward modeling its behaviour: 725 Physics and Chemistry of the Earth, 9, 785-791.

Gao, F., A. Levander, R. Pratt, C. Zelt, and G. Fradelizio, 2006, Waveform 727 tomography at a groundwater contamination site: VSP-surface data set: 728 Geophysics, 71, no. 1, H1-H11, doi:10.1190/1.2159049.

2007, Waveform tomography at a groundwater contamination 730 site: Surface reflection data: Geophysics, 72, no. 5, G45-G55, doi: 731 $10.1190 / 1.2752744$.

Gélis, C., 2005, Inversion des formes d'onde élastiques dans le domaine espace-fréquence en deux dimensions: Application à la caractérisation de la subsurface dans le cadre de la détection de cavités souterraines: Ph.D. dissertation, Université de Nice-Sophia-Antipolis.

Gélis, C., J. Virieux, and G. Grandjean, 2007, Two-dimensional elastic 737 waveform inversion using Born and Rytov formulations in the fre- 738 quency domain: Geophysical Journal International, 168, 605-633, 739 doi:10.1111/j.1365-246X.2006.03135.x.

Grandjean, G., and A. Bitri, 2006, 2M-SASW: Multifold multichanned 741 seismic inversion of local dispersion of Rayleigh waves in laterally het- 742 erogenous subsurfaces: Application to the Super-Sauze earthflow, 743 France: Near Surface Geophysics, 4, 367-375.

Grandjean, G., C. Pennetier, A. Bitri, O. Méric, and J. Malet, 2006, Carac- 745 térisation de la structure interne et de l'état hydrique de glissements 746 argilo-marneux par tomographie géophysique: l'exemple du glissement- 747 coulée de Super-Sauze (Alpes du Sud, France): Comptes Rendus Geo- 748 sciences, 338, 587-595, doi:10.1016/j.crte.2006.03.013.

Grandjean, G., and D. Leparoux, 2004, The potential of seismic methods 750 for detecting cavities and buried objects: Experiments at a test site: 75 Journal of Applied Geophysics, 56, no. 2, 93-106, doi:10.1016/ 752 j.jappgeo.2004.04.004

Grandjean, G., and S. Sage, 2004, JaTS: A fully portable seismic tomogra- 754 phy software based on Fresnel wavepaths and a probabilistic reconstruc- 755 tion approach: Computers \& Geosciences, 30, 925-935, doi:10.1016/ 756 j.cageo.2004.06.009.

Hermann, R., 1991, Surf: Surface wave inversion program: Saint Louis 758 University.

Lai, C. G., 1998, Simultaneous inversion of Rayleigh phase velocity and 760 attenuation for near-surface site characterization: Ph.D. dissertation, 761 Georgia Institute of Technology.

Moczo, P., J. O. A. Robertsson, and L. Eisner, 2007, The finite-difference 763 time-domain method for modeling of seismic wave propagation: 764 Advances in Geophysics, 48, 421-516, doi:10.1016/S0065-2687(06) 765 48008-0.

MUMPS Team, 1999. MUMPS - MUltifrontal Massively Parallel Solver 767 users' guide, v. 4.9.2: Ecole Nationale Supérieure d'Electrotechnique, 768 d'Electronique, d'Informatique, d'Hydraulique et des Télécommunica- 769 tions (ENSEEIHT), accessed 8 January 2011, http://mumps.enseeiht. fr/ 770 doc/userguide_4.9.2.pdf. 


\section{PROOF COPY [2010-0066] 057103GPY}

Nazarian, S., and I. K. H. Stokoe, 1984, In situ shear wave velocity from spectral analysis of surface waves: Near Surface Geophysics, 3, 31-38.

1986, Use of surface waves in pavement evaluation: Transportation Research Record, 1070, 132-144.

Nocedal, J., and S. J. Wright, 1999, Numerical optimization: SpringerVerlag New York.

Operto, S., J. Virieux, J. X. Dessa, and G. Pascal, 2006, Crustal seismic imaging from multifold ocean bottom seismometers data by frequency domain full waveform tomography: Application to the eastern Nankai trough: Journal of Geophysical Research, Solid Earth, 111, B9, B09306, doi:10.1029/2005JB003835.

Park, C., R. Miller, and J. Xia, 1998, Imaging dispersion curves of surface waves on a multichannel record: 68th Annual International Meeting, SEG, Expanded Abstracts, 1377-1380.

, 1999, Multichannel analysis of surface waves: Geophysics, 64, 800-808, doi:10.1190/1.1444590.

Plessix, R.-E., 2006, A review of the adjoint-state method for computing the gradient of a functional with geophysical applications: Geophysical Journal International, 167, 495-503, doi:10.1111/j.1365-246X.2006. 02978.x.

Podvin, P., and I. Lecomte, 1991, Finite difference computation of traveltimes in very contrasted velocity model: A massively parallel approach and its associated tools: Geophysical Journal International, 105, no. 1, 271-284, doi:10.1111/j.1365-246X.1991.tb03461.x.

Pratt, R. G., 1999, Seismic waveform inversion in the frequency domain, Part I: Theory and verification in a physic scale model: Geophysics, 64, 888-901, doi:10.1190/1.1444597

Pratt, R. G., C. Shin, and G. J. Hicks, 1998, Gauss-Newton and full Newton methods in frequency-space seismic waveform inversion: Geophysical Journal International, 133, no. 2, 341-362, doi:10.1046/j.1365246X.1998.00498.x

Pratt, R. G., and R. M. Shipp, 1999, Seismic waveform inversion in the frequency domain, Part II: Fault delineation in sediments using crosshole data: Geophysics, 64, 902-914, doi:10.1190/1.1444598.

Ravaut, C., S. Operto, L. Improta, J. Virieux, A. Herrero, and P. dell'Aversana, 2004, Multiscale imaging of complex structures from multifold wide-aperture seismic data by frequency-domain full-wavefield tomography: Application to a thrust belt: Geophysical Journal International, 159, 1032-1056, doi:10.1111/j.1365-246X.2004.02442.x.

Rix, G. J., C. G. Lai, and S. Foti, 2001, Simultaneous measurement of surface wave disper-sion and attenuation curves: Geotechnical Testing Journal, 24, no. 4, 350-358, doi:10.1520/GTJ11132J.

Romdhane, A., G. Grandjean, A. Bitri, and F. Réjiba, 2008, Inversion of surface waves dispersion in complex structures: 21st Symposium on the Application of Geophysics to Environmental and Engineering Problems (SAGEEP), Poster Session.

2009, Full waveform inversion of seismic data for 2D shallow structures imagery: 71st Conference and Technical Meeting, EAGE, Extended Abstracts, P002.

Saenger, E. H., N. Gold, and S. A. Shapiro, 2000, Modeling the propagation of elastic waves using a modified finite-difference grid: Wave Motion, 31, no. 1, 77-92, doi:10.1016/S0165-2125(99)00023-2.

Sheng, J., A. Leeds, M. Buddensiek, and G. T. Schuster, 2006, Early arrival waveform tomography on near-surface refraction data: Geophysics, 71, no. 4, U47-U57, doi:10.1190/1.2210969.

Shiann-Jong, L., D. Komatitsch, B.-S. Huang, and J. Tromp, 2009, Effects of topography on seismic-wave propagation: An example from northern Taiwan: Bulletin of the Seismological Society of America, 99, no. 1, 314-325, doi:10.1785/012008t020.

Shin, C., S. Jang, and D.-J. Min, 2001, Improved amplitude preservation for prestack depth migration by inverse scattering theory: Geophysical Prospecting, 49, 592-606, doi:10.1046/j.1365-2478.2001.00279.x.
Shin, C., D.-J. Min, K. J. Marfurt, H. Y. Lim, D. Yang, Y. Cha, S. Ko, K. 834 Yoon, T. Ha, and S. Hong, 2002, Traveltime and amplitude calculations 835 using the damped wave solution: Geophysics, 67, 1637-1647, doi: 836 10.1190/1.1512811.

Sirgue, L., and R. G. Pratt, 2004, Efficient waveform inversion and imag- 838 ing: A strategy for selecting temporal frequencies: Geophysics, 69, 839 231-248, doi:10.1190/1.1649391

Smithyman, B., R. G. Pratt, J. Hayles, and R. Wittebolle, 2009, Detecting 84 near-surface objects with seismic waveform tomography: Geophysics, 842 74, no. 6, WCC119-WCC127, doi:10.1190/1.3223313.

Socco, L., and C. Strobia, 2004, Surface-wave method for near-surface 844 characterization: A tutorial: Near Surface Geophysics, 2, 165-185. 845

Sourbier, F., S. Operto, J. Virieux, P. Amestoy, and J.-Y. L'Excellent, 846 2009, FWT2D: A massively parallel program for frequency-domain 847 full-waveform tomography of wide-aperture seismic data - Part 1: 848 Algorithm: Computers \& Geosciences, 35, 487-495, doi:10.1016/ 849 j.cageo.2008.04.013.

Stokoe, I. K. H., and S. Nazarian, 1985, Use of Rayleigh wave in liquefac- 851 tion studies: Proceedings of the Measurement and Use of Shear Wave 852 Velocity for Evaluating Dynamic Soil Properties, American Society of 853 Civil Engineers, 1-17.

Stokoe, I. K. H., S. Nazarian, G. J. Rix, I. Sanchez-Salinero, J. Sheu, and 855 Y. Mok, 1988, In situ seismic testing of hard-to-sample soils by surface 856 wave method: American Society of Civil Engineers Special Publication 857 20, 264-278.

Taillandier, C., M. Noble, H. Chauris, and H. Calandra, 2009, First-arrival 859 traveltime tomography based on the adjoint-state method: Geophysics, 860 74, no. 6, WCB1-WCB10, doi:10.1190/1.3250266.

Tarantola, A., 1984, Linearized inversion of seismic reflection data: Geo- 862 physical Prospecting, 32, 998-1015, doi:10.1111/j.1365-2478.1984. 863 tb00751.x.

1987, Inverse problem theory: Methods for data fitting and model 865 parameter estimation: Elsevier Scientific Publ. Co., Inc. 866

Toverud, T., and B. Ursin, 2005, Comparison of seismic attenuation mod- 867 els using zero-offset vertical seismic profiling (VSP) data: Geophysics, 868 70, no. 2, F17-F25, doi:10.1190/1.1884827. 869 van der Sluis, A., and H. A. van der Vorst, 1987, Numerical solution 870 of large, sparse linear algebraic systems arising from tomographic 871 problems, in G. Nolet, ed., Seismic tomography, with applications 872 in global seismology and exploration geophysics: Reidel Publishing, 873 49-83.

Vidale, D., 1988, Finite-difference calculation of travel time: Bulletin of 875 the Seismological Society of America, 78, 2062-2076. 876

Viktorov, I. A., 1965, Rayleigh and Lamb waves: Physical theory applica- 877 tions: Plenum Press.

Vinje, V., E. Iversen, K. Astebøl, and H. Gjøystdal, 1996a, Estimation of 879 multivalued arrivals in 3D models using wavefront construction - Part 880 I: Geophysical Prospecting, 44, 819-842, doi:10.1111/j.1365-2478. 88 1996.tb00175.x.

1996b, Estimation of multivalued arrivals in 3D models using 883 wavefront construction - Part II: Tracing and interpolation: Geophysi- 884 cal Prospecting, 44, 843-858, doi:10.1111/j.1365-2478.1996. 885 tb00176.x.

Vinje, V., E. Iversen, and H. Gjøystdal, 1993, Traveltime and amplitude 887 estimation using wavefront construction: Geophysics, 58, 1157-1166, 888 doi:10.1190/1.1443499. 889

Virieux, J., and S. Operto, 2009, An overview of full-waveform inversion 890 in exploration geophysics: Geophysics, 74, no. 6, WCC1-WCC26, 891 doi:10.1190/1.3238367.

Xia, J., R. Miller, and C. Park, 1999, Estimation of near-surface shear- 893 wave velocity by inversion of Rayleigh waves: Geophysics, 64, 691- 894 700, doi: 10.1190/1.1444578. 